\title{
Flow-Based Feasibility Test of Linear Interference Alignment with Arbitrary Interference Topology
}

\author{
Peng-Jun Wan*, Fahad Al-Dhelaan ${ }^{\dagger}$, Sai Ji ${ }^{\ddagger}$, Lei $\mathrm{Wang}^{\S}$, and Ophir Frieder $\llbracket$ \\ * Department of Computer Science, Illinois Institute of Technology, wan@cs.iit.edu \\ $\dagger$ Department of Computer Science, Illinois Institute of Technology, faldhela@iit.edu \\ $\ddagger$ College of Computer And Software, Nanjing University of Information Science And Technology, jisai@ @uist.edu.cn \\ $\S$ School of Software, Dalian University of Technology, lei.wang @ieee.org \\ I Department of Computer Science, Georgetown University, ophir@ir.cs.georgetown.edu
}

\begin{abstract}
Linear interference alignment (LIA) is one of the key interference mitigation techniques to enhance the wireless MIMO network capacity. The generic LIA feasibility amounts to whether or not a well-structured random matrix with entries drawn from a continuous distribution has full row-rank almost surely. Recently, a randomized algebraic test of feasibility was proposed in the literature. It is a pseudo-polynomial boundederror probabilistic algorithm in nature, and has intrinsic limitations of requiring an inordinate amount of running time and memory even for a moderate sized input and being prone to round-off errors in floating-point computations. This paper presents necessary conditions and sufficient conditions of the generic LIA feasibility and develops fast and robust tests of them based on network flow. In certain settings, these conditions are both necessary and sufficient, and their flow-based tests yield efficient algorithm for feasibility test.
\end{abstract}

\section{INTRODUCTION}

Linear interference alignment (LIA) [4], [12], [13], [16] with beam-forming (BF) and zero-forcing (ZF) is one of the key interference mitigation techniques to enhance the wireless multiple-input multiple-output (MIMO) network capacity. The generic LIA feasibility, or simply the feasibility in short, amounts to the solvability of a system of bilinear equations with coefficients drawn independently from a continuous distribution [1], [7], [9]. It has been intensively studied w.r.t. a complete interference topology [3], [15], [18], [19], [20], [23], [29] as well as an arbitrary interference topology [10], [11], [14], [21], [22], [28], and was settled by González et al. [8] very recently in arbitrary settings: it reduces to check whether or not a well-structured random matrix with entries drawn from a continuous distribution has full row-rank almost surely. Based on such reduction, González et al. [8] proposed a randomized algebraic test of feasibility by determining the ranks of very large random matrices. This randomized algebraic test is a bounded-error probabilistic algorithm in nature: it always gives a correct answer to an infeasible instance, but may give a false answer otherwise with an error probability decreasing exponentially with the number of test rounds. However, it was noted in [8] that any algebraic test by determining the rank of a very large matrix has intrinsic limitations of requiring an inordinate amount of running time and memory even for a moderate sized input and being prone to round-off errors in floating-point computations. As articulated in Section II, this randomized algebraic test is pseudo-polynomial in general.

Prior to the randomized algebraic test [8], combinatorial tests of feasibility w.r.t. a complete interference topology (i.e., single interference domain) by counting the number of (free) $\mathrm{BF} / \mathrm{ZF}$ variables involved in all or some subsets of LIA equations have been proposed in [3], [20], [23], [29]. It was first observed in [29] that a necessary condition of feasibility is that every subset of LIA equations must involve BF/ZF variables at least as many as the number of equations in that subset. Such necessary condition is termed properness in [29]. As the properness is defined by an exponential number of constraints, a prevailing criticism [8], [29] on the test of properness is that it is computationally cumbersome and demanding. A weak (and simple) variant of the properness with much fewer but still exponential number of constraints is also introduced in [3], [20], [23]. In some special settings, the weak variant of the properness, and hence the properness itself, also turns out to be a sufficient condition for feasibility and can be easily verified. But in general, due to the exponential number of constraints, the tests of properness and its weak variant have been limited to small "toy" examples so far, and a common belief is that properness and its weak variant are hard to trace in both analysis and practice [23].

Despite of the common belief on the intractability of properness and its weak variant, combinatorial test appears to be the most natural fit to the test of feasibility based on the following observation. Consider a finite set $X$ of continuous-valued random variables, and a random matrix $\mathbf{M}$ in which each of its entry is either a fixed constant or a random variable in $X$. From the Leibniz formula [17], the determinant of each square sub-matrix of $\mathbf{M}$ is a polynomial of $X$, and hence is either zero for all values of $X$ or is non-zero out of a zero-measured set by the Fundamental Theorem of Algebra. Accordingly, $\mathbf{M}$ either has deficient row-rank certainly or has full row-rank almost surely; and whichever to occur is determined only on the structural, rather than numerical, dependence of $\mathbf{M}$ on $X$. Thus, feasibility is combinatorial in nature, and its test via a combinatorial method is a natural option. The intractability of the exponentially exhaustive counting does not pronounce the genetic inviability of the combinatorial tests. Instead, it only serves as a call for more efficient methods for combinatorial tests than the naive exhaustive counting-which should be absolutely avoided.

Inspired by the above observation and motivated by the application of LIA in general multihop wireless MIMO networks, this paper aims at developing efficient and robust combinatorial tests of feasibility w.r.t. an arbitrary interference topology. To this end, we first extend the concepts of properness and 
weak properness to the setting with arbitrary interference topology, and then develop their combinatorial tests based on network flow. The flow-based tests are not only efficient and robust, but also applicable to arbitrary settings (for any number of users, antennas and streams per user, and for any interference topology). As properness and weak properness are necessary conditions for feasibility, our flow-based tests can quickly detect the infeasibility of improper or weakly improper instances. A by-product of the flow-based tests is the discovery of the equivalence of properness and weaker properness in the case of uniform streams. We further introduce the concept of strong properness, which is a variant of the properness stronger enough to be a sufficient condition for feasibility. While the test of the strong properness seems to be intractable in general, an efficient flow-based test is developed in this paper for the case of uniform streams. Thus, this flowbased test can quickly detect the feasibility of a strongly proper instance with uniform streams. Furthermore, for the special case of uniform number of streams which either divides the number of transmitting antennas of every user or divides the number of receiving antennas of every user, our flow-based tests imply the equivalence of strong properness, properness, weak properness, and feasibility. In particular, the feasibility of the single-stream instances can be efficiently decided.

The paper is organized as follows. Section II briefly reviews LIA in arbitrary wireless MIMO networks. Section III defines the variants of properness and presents the relations among them and the feasibility. Section IV introduces an reduction on the interference topology which preserves all four properties and the concept of irreducible interference topology. Section V presents the flow-based tests of weak properness, properness and strong properness w.r.t. an irreducible interference topology and their implications on feasibility test. Finally, Section VI summarizes this paper and discusses on future works.

We conclude this section with the standard notations adopted in this paper. The plain symbol $a$ and the bold symbols $\mathbf{a}$ and $\mathbf{A}$ represent scalar, vector, matrix respectively. The blackboard bold symbols $\mathbb{N}, \mathbb{Z}$ and $\mathbb{C}$ denote the set of natural numbers, integers and complex numbers, respectively. For any $k \in \mathbb{N},[k]$ denotes the set $\{1,2, \cdots, k\}$ consisting of the first $k$ positive integers. Suppose that $\mathbf{A}$ is an $m \times n$ matrix. The transpose of $\mathbf{A}$ is denoted by $\mathbf{A}^{\top}$; for any $i \in[m]$ and $j \in[n]$, $\mathbf{A}_{i}$. denote the $i$-th row vector of $\mathbf{A}, \mathbf{A} . j$ denote the $j$-th column vector of $\mathbf{A}$, and $a_{i j}$ denotes the entry of $\mathbf{A}$ in the $i$-th row and the $j$-th column.

\section{GENERIC LIA FEASIBILITY}

Consider a set $[\ell]$ of users (or links, requests, interchangeably) in a wireless MIMO network. Each user $i \in[\ell]$, its transmitting node has $M_{i}$, its receiving node has $N_{i} \geq 1$ antennas, and it has to send $d_{i}$ independent data streams where $1 \leq d_{i} \leq \min \left\{M_{i}, N_{i}\right\}$. The set of triples

$$
\mathcal{L}=\left\{\left(M_{i}, N_{i}, d_{i}\right): i \in[\ell]\right\}
$$

is referred to as an instance of users. $\mathcal{L}$ is said to be symmetric if $\left(M_{i}, N_{i}, d_{i}\right)=(M, N, d)$ for all $i \in[\ell]$; in this case we simply denote $\mathcal{L}=(M, N, d)^{\ell} . \mathcal{L}$ is said to have uniform steams if $d_{i}=d$ for all $i \in[\ell]$. In particular, if $d_{i}=1$ for all $i \in[\ell], \mathcal{L}$ is said to be a single-stream instance.
The interference topology of an instance $\mathcal{L}$ is specified by a set $\mathcal{E}$ of ordered pairs of distinct users. A pair $(j, k) \in \mathcal{E}$ represents the generic MIMO channel state from the transmitter of $j$ to the receiver of $k$ is non-zero and thus indicates the interference from $j$ to $k$. In general, the interference topology may be asymmetric, i.e., it is possible that $(j, k) \in \mathcal{E}$ but $(k, j) \notin \mathcal{E}$. If $\mathcal{E}$ consists of all possible $(\ell(\ell-1))$ pairs, then the interference topology is said to be complete. The first projection of $\mathcal{E}$ is the set

$$
\mathcal{E}_{T}=\{j \in[\ell]: \exists k \in[\ell],(j, k) \in \mathcal{E}\},
$$

and the second projection of $\mathcal{E}$ is the set

$$
\mathcal{E}_{R}=\{k \in[\ell]: \exists j \in[\ell],(j, k) \in \mathcal{E}\} .
$$

In other words, $\mathcal{E}_{T}$ consists of the users which provoke interference to at least one other user, whereas $\mathcal{E}_{R}$ consists of the users which suffer interference from at least one other user. For any user $j \in[\ell]$, the set of users interfered by $j$ w.r.t. $\mathcal{E}$ is the set

$$
\Gamma_{\mathcal{E}}^{\text {out }}(j)=\{k:(j, k) \in \mathcal{E}\} ;
$$

in addition, $\Gamma_{\mathcal{E}}^{\text {out }}[j]$ denotes $\Gamma_{\mathcal{E}}^{o u t}(j) \cup\{j\}$. For any user $k \in$ $[\ell]$, the set of users interfering $k$ w.r.t. $\mathcal{E}$ is the set

$$
\Gamma_{\mathcal{E}}^{i n}(k)=\{j:(j, k) \in \mathcal{E}\} ;
$$

in addition, $\Gamma_{\mathcal{E}}^{i n}[j]$ denotes $\Gamma_{\mathcal{E}}^{i n}(j) \cup\{j\}$. The subscript $\mathcal{E}$ may be omitted if it is clear from the context. For any user $j \in[\ell]$, let $\mathcal{E}-(j, \cdot)$ be the interference topology obtained from $\mathcal{E}$ by removing all pairs $(j, k) \in \mathcal{E}$; and for any user $k \in[\ell]$, let $\mathcal{E}-(\cdot, k)$ be the interference topology obtained from $\mathcal{E}$ by removing all pairs $(j, k) \in \mathcal{E}$.

The generic LIA feasibility, or simply feasibility, of $\mathcal{L}$ w.r.t. an interference topology $\mathcal{E}$ was beautifully settled by [8]. Suppose that

$$
\begin{aligned}
& \left\{\mathbf{A}^{(j, k)} \in \mathbb{C}^{d_{k} \times\left(M_{j}-d_{j}\right)}:(j, k) \in \mathcal{E}\right\} \cup \\
& \left\{\mathbf{B}^{(j, k)} \in \mathbb{C}^{d_{j} \times\left(N_{k}-d_{k}\right)}:(j, k) \in \mathcal{E}\right\}
\end{aligned}
$$

is a set of $2|\mathcal{E}|$ random matrics whose entries are drawn independently from continuous distributions. These (random) matrices define a (random) linear mapping

$$
\begin{aligned}
\mathbf{F} & \left(\prod_{j \in \mathcal{E}_{T}} \mathbb{C}^{\left(M_{j}-d_{j}\right) \times d_{j}}\right) \times\left(\prod_{k \in \mathcal{E}_{R}} \mathbb{C}^{\left(N_{k}-d_{k}\right) \times d_{k}}\right) \rightarrow \\
& \prod_{(j, k) \in \mathcal{E}} \mathbb{C}^{d_{j} \times d_{k}}
\end{aligned}
$$

as follows. For each user $j \in \mathcal{E}_{T}$, let $\mathbf{U}^{(j)} \in \mathbb{C}^{\left(M_{j}-d_{j}\right) \times d_{j}}$ be the matrix of its (free) $\mathrm{BF}$ variables; for each user $k \in \mathcal{E}_{R}$, let $\mathbf{V}^{(k)} \in \mathbb{C}^{d_{k} \times\left(M_{j}-d_{j}\right)}$ be the matrix of its (free) $\mathrm{ZF}$ variables. The image of these $\mathrm{BF} / \mathrm{ZF}$ variables is

$$
\left\{\mathbf{F}^{(j, k)}=\left(\mathbf{A}^{(j, k)} \mathbf{U}^{(j)}\right)^{\top}+\mathbf{B}^{(j, k)} \mathbf{V}^{(k)}:(j, k) \in \mathcal{E}\right\} .
$$

More specifically, $\mathbf{F}$ consists of the following $\sum_{(j, k) \in \mathcal{E}} d_{j} d_{k}$ equations

$$
\bigcup_{(j, k) \in \mathcal{E}}\left\{f_{p q}^{(j, k)}=\mathbf{A}_{q \cdot}^{(j, k)} \mathbf{U}_{\cdot p}^{(j)}+\mathbf{B}_{p}^{(j, k)} \mathbf{V}_{\cdot q}^{(k)}: p \in\left[d_{j}\right], q \in\left[d_{k}\right]\right\}
$$


Theorem 2.1: $\mathcal{L}$ is feasible w.r.t. $\mathcal{E}$ if and only if the mapping $\mathbf{F}$ is surjective almost surely.

To facilitate the test of (almost sure) surjectivity of the linear mapping $\mathbf{F}$, a matrix form $\mathbf{\Phi} \mathbf{w}$ of $\mathbf{F}$ is adopted, where $\mathbf{w}$ is a column vector consisting of all $\mathrm{BF}$ variables

$$
\bigcup_{j \in \mathcal{E}_{T}}\left\{u_{m p}^{(j)}: m \in\left[M_{j}-d_{j}\right], p \in\left[d_{j}\right]\right\}
$$

and all ZF variables

$$
\bigcup_{k \in \mathcal{E}_{R}}\left\{v_{n q}^{(k)}: n \in\left[N_{k}-d_{k}\right], q \in\left[d_{k}\right]\right\},
$$

and $\Phi$ is a matrix of dimensions

$$
\left(\sum_{(j, k) \in \mathcal{E}} d_{j} d_{k}\right) \times\left(\sum_{j \in \mathcal{E}_{T}}\left(M_{j}-d_{j}\right) d_{j}+\sum_{k \in \mathcal{E}_{R}}\left(N_{k}-d_{k}\right) d_{k}\right) .
$$

The matrix $\boldsymbol{\Phi}$ is uniquely determined by both the ordering of the BF/ZF variables in (2) and (3) and the ordering of the equations in (1). Accordingly, each row of $\Phi$ is indexed by an (unique) equation in (1) and each column of $\boldsymbol{\Phi}$ is indexed by an (unique) BF/ZF variable in (2) and (3). Note that all instantiations of $\boldsymbol{\Phi}$ have the same row-rank, and checking the feasibility of IA then reduces to check whether an instantiation of $\Phi$ has full row-rank or not. Thus, any instantiation of $\Phi$ is referred to as a LIA matrix of $\mathcal{L}$ w.r.t. $\mathcal{E}$.

Inspired by Shub and Smale's construction [2], [25] for polynomial system solving, González et al. [8] proposed a randomized algebraic test of the generic LIA feasibility. Let

$$
h=\sum_{(j, k) \in \mathcal{E}}\left(\left(M_{j}-d_{j}\right) d_{j}+\left(N_{k}-d_{k}\right) d_{k}\right) .
$$

Each round of the test chooses $\mathbf{A}^{(j, k)}, \mathbf{B}^{(j, k)}$ whose entries are selected (i.i.d. uniformly) from the set

$$
\{a+\sqrt{-1} b: 0 \leq a, b \leq h ; a, b \in \mathbb{Z}\} .
$$

and checks if $\mathbf{F}$ is surjective using exact linear algebra procedures (such as the ones available in [5], [6]). Then,

- If $\mathcal{L}$ is infeasible w.r.t. $\mathcal{E}$, the test always answers infeasible.

- If $\mathcal{L}$ is feasible w.r.t. $\mathcal{E}$, the test may answer infeasible with a probability of at most $1 / 4$.

The use of above test is very simple: if on a given choice of $\mathbf{A}^{(j, k)}, \mathbf{B}^{(j, k)}$ the test answers feasible then $\mathcal{L}$ is feasible w.r.t. $\mathcal{E}$. If the test is run $k$ times and its answer is infeasible for all $k$ tests, then the algorithm can conclude that $\mathcal{L}$ is infeasible w.r.t. $\mathcal{E}$ which may err with probability only at most $4^{-k}$. Now, let us take a careful look at the running time. The inputs have bit length of

$$
\Theta\left(\ell^{2}+\ell \max \left(1+\log _{2} \max _{j \in[\ell]}\left\{M_{j}, N_{j}\right\}\right)\right) .
$$

However, the running time is poly $\left(\ell, \max _{j \in[\ell]}\left\{M_{j}, N_{j}\right\}\right)$, which is is only pseudo-polynomial unless

$$
\max _{j \in[\ell]}\left\{M_{j}, N_{j}\right\}=\operatorname{poly}(\ell) .
$$

It was noted in [8] that the randomized test lacks scalability in both running time and memory requirement, and any algebraic test by determining the rank of a very large matrix has intrinsic limitations of being very computationally intensive and prone to round-off errors in floating-point computations.

\section{VARIANTS OF PROPERNESS}

Consider an instance $\mathcal{L}$ of users and an interference topology $\mathcal{E}$ of $\mathcal{L}$. $\mathcal{L}$ is said to be proper w.r.t. $\mathcal{E}$ if for any subset of equations in (1) the number of BF/ZF variables involved is at least equal to the number of equations in that subset. While this definition of properness has $2^{\sum_{(j, k) \in \mathcal{E}} d_{j} d_{k}}$ constraints, we introduce an equivalent graph-theoretic characterization. We construct a bipartite graph $G$ on equations and $\mathrm{BF} / \mathrm{ZF}$ variables as follows. The first class of the vertices consists of all equations in (1); the second class of the vertices consists of all BF variables in (2) and all ZF variables in (3). There is an edge between an equation and a variable if and only if the equation contains that variable. Then, the properness is characterized by the following variant of Hall's condition: for every subset of equations, the number of their neighbors in $G$ is at least equal to the number of equations in that subset. By a generalized Hall's Theorem (cf. Theorem 16.7 in [24]), the system is proper if and only if there exists a complete matching of all equations $G$.

For any $J \subseteq \mathcal{E}_{T}$ and $K \subseteq \mathcal{E}_{R}$, let

$$
\mathcal{E}[J, K]=\{(j, k) \in \mathcal{E} \mid j \in J, k \in K\} .
$$

$\mathcal{L}$ is said to be weakly proper w.r.t. $\mathcal{E}$ if for any $J \subseteq \mathcal{E}_{T}$ and $K \subseteq \mathcal{E}_{R}$, the number of variables appearing in the subset of equations

$$
\bigcup_{(j, k) \in \mathcal{E}[J, K]}\left\{f_{p q}^{(j, k)}: p \in\left[d_{j}\right], q \in\left[d_{k}\right]\right\} .
$$

is at least equal to the number of equations in this subset, that is

$$
\sum_{j \in J} d_{j}\left(M_{j}-d_{j}\right)+\sum_{k \in K} d_{k}\left(N_{k}-d_{k}\right) \geq \sum_{(j, k) \in \mathcal{E}[J, K]} d_{k} d_{j} .
$$

The total number of constraints is $2^{\left|\mathcal{E}_{T}\right|+\left|\mathcal{E}_{R}\right|}$, which is much fewer but still exponential.

The properness (and consequently, weak properness) is only a necessary condition of feasibility. Indeed, the symmetric instance $(3,3,2)^{2}$ with a complete interference topology is proper but is infeasible (cf. Example 17 in [29]). Next, we introduce a strong variant of properness which is a sufficient condition of feasibility. Let $\mathcal{M}$ be a complete matching of the equations with the variables. $\mathcal{M}$ is said to be Tx-strong if each group of equations

$$
\mathbf{F}_{\cdot q}^{(j, k)}=\left\{f_{p q}^{(j, k)}: p \in\left[d_{j}\right]\right\}
$$

are either all matched with the BF variables or all matched with the ZF variables in $\mathcal{M}$. Similarly, $\mathcal{M}$ is said to be $R x$-strong if each group of equations

$$
\mathbf{F}_{p \cdot}^{(j, k)}=\left\{f_{p q}^{(j, k)}: q \in\left[d_{k}\right]\right\}
$$

are either all matched with the BF variables or all matched with the $\mathrm{ZF}$ variables in $\mathcal{M}$. In general, $\mathcal{M}$ is said to be strong if it is either Tx-strong or Rx-strong. The instance $\mathcal{L}$ is said to be $T x$-strongly proper (resp., $R x$-strongly proper) w.r.t. $\mathcal{E}$ if 
it admits a Tx-strong (resp., Rx-strong) complete matching of equations with the variables. In general, $\mathcal{L}$ is said to be strongly proper if it is either $\mathrm{Tx}$-strongly proper or $\mathrm{Rx}$-strongly proper.

Theorem 3.1: If $\mathcal{L}$ is strongly proper w.r.t. $\mathcal{E}$, then $\mathcal{L}$ is feasible w.r.t. $\mathcal{E}$.

The proof of the above theorem is omitted here due to the space limitation. In summary, the following sequence of implications holds:

$$
\begin{aligned}
& \text { strong properness } \Rightarrow \text { feasibility } \Rightarrow \text { properness } \\
& \Rightarrow \text { weak properness. }
\end{aligned}
$$

\section{REDUCED INTERFERENCE TOPOLOGY}

Consider an instance $\mathcal{L}$ of users and an interference topology $\mathcal{E}$ of $\mathcal{L}$. A user $j$ is said to be $T x$-superfluous w.r.t. $\mathcal{E}$ if $M_{j} \geq \sum_{k \in \Gamma_{\varepsilon}^{o u t}[j]} d_{k}$. A user $k$ is said to be Rx-superfluous w.r.t. $\mathcal{E}$ if $N_{k}^{\mathcal{E}} \geq \sum_{j \in \Gamma_{\mathcal{E}}^{i n}[k]} d_{j}$. If a user $j$ is Tx-superfluous w.r.t. $\mathcal{E}$, then $\mathcal{E}-(j, \cdot)$ is referred to as a $T x$-reduction of $\mathcal{E}$. If a user $k \in \mathcal{E}_{R}$ is $\mathrm{Rx}$-superfluous w.r.t. $\mathcal{E}$, then the $\mathcal{E}-(\cdot, k)$ is referred to as a $R x$-reduction of $\mathcal{E}$. An interference topology $\mathcal{E}^{\prime}$ of $\mathcal{L}$ is said to be a reduction of $\mathcal{E}$ if it is either a Txreduction of $\mathcal{E}$ or a Rx-reduction of $\mathcal{E}$. The next theorem below shows that the reduction operation does not change feasibility, properness, strong properness, and weak properness.

Theorem 4.1: Suppose that $\mathcal{E}^{\prime}$ is a reduction of $\mathcal{E}$. Then, $\mathcal{L}$ is feasible (respectively, proper, strongly proper, weakly proper) w.r.t. $\mathcal{E}$ iff $\mathcal{L}$ is feasible (respectively, proper, strongly proper, weakly proper) w.r.t. $\mathcal{E}^{\prime}$.

The proof of the above theorem is omitted here due to the space limitation.

An interference topology $\mathcal{E}^{\prime}$ of $\mathcal{L}$ is said to be irreducible if no user is Tx-superfluous w.r.t. $\mathcal{E}^{\prime}$ or Rx-superfluous w.r.t. $\mathcal{E}^{\prime}$. An irreducible interference topology $\mathcal{E}^{\prime}$ of $\mathcal{L}$ can be obtained by $\mathcal{E}$ by a (arbitrary) sequence of reductions. Theorem $4.1 \mathrm{im}-$ plies that $\mathcal{L}$ has the same feasibility (respectively, properness, strong properness, and weak properness) w.r.t. $\mathcal{E}$ as w.r.t. $\mathcal{E}^{\prime}$. Thus, we assume that the interference topology $\mathcal{E}$ is irreducible subsequently.

\section{Network-Flow Based Tests}

In this section, we consider an instance $\mathcal{L}$ of users and an irreducible interference topology $\mathcal{E}$ of $\mathcal{L}$, and present flow-based tests of weak properness, properness, and strong properness and their implications on feasibility test.

\section{A. Weak Properness}

In this subsection, we present flow-based test of weak properness. We construct two flow networks $\mathcal{N}_{1}$ and $\mathcal{N}_{2}$ as follows. Let $s$ and $t$ be two distinct source and sink vertices. Both networks have the same vertex set

$$
\{s, t\} \cup\left\{\mathbf{U}^{(j)}: j \in \mathcal{E}_{T}\right\} \cup\left\{\mathbf{V}^{(k)}: k \in \mathcal{E}_{R}\right\},
$$

and the same arc set

$$
\begin{aligned}
& \left\{\left(s, \mathbf{U}^{(j)}\right): j \in \mathcal{E}_{T}\right\} \cup\left\{\left(\mathbf{V}^{(k)}, t\right): k \in \mathcal{E}_{R}\right\} \cup \\
& \left\{\left(\mathbf{U}^{(j)}, \mathbf{V}^{(k)}\right):(j, k) \in \mathcal{E}\right\}
\end{aligned}
$$

The arc capacities in these two flow networks are given in Table I. Both flow networks have $O(\ell)$ vertices and $O(|\mathcal{E}|+\ell)$ arcs. Thus, a maximum $s$ - $t$ flow can be computed in time $\widetilde{O}(\ell(|\mathcal{E}|+\ell)$ ) (cf. Section $10.8 \mathrm{~b}$ in [24]). By the celebrated max-flow min-cut theorem, the maximum $s$ - $t$ flow in $\mathcal{N}_{1}$ has value at most $\sum_{j \in \mathcal{E}_{T}} d_{j}\left(\sum_{k \in \Gamma^{\text {out }[j]}} d_{k}-M_{j}\right)$, and the maximum $s$ - $t$ flow in $\mathcal{N}_{2}$ has value at most $\sum_{k \in \mathcal{E}_{R}} d_{k}\left(\sum_{j \in \Gamma^{i n}[k]} d_{j}-N_{k}\right)$. The theorem below shows that these upper bounds are achievable if and only if $\mathcal{L}$ is weakly proper w.r.t. $\mathcal{E}$.

\begin{tabular}{|c||c|c|}
\hline & $\mathcal{N}_{1}$ & $\mathcal{N}_{2}$ \\
\hline \hline$\left(s, \mathbf{U}^{(j)}\right)$ & $d_{j}\left(\sum_{k \in \Gamma^{\text {out }}[j]} d_{k}-M_{j}\right)$ & $d_{j}\left(M_{j}-d_{j}\right)$ \\
\hline$\left(\mathbf{V}^{(k)}, t\right)$ & $d_{k}\left(N_{k}-d_{k}\right)$ & $d_{k}\left(\sum_{j \in \Gamma^{i n}[k]} d_{j}-N_{k}\right)$ \\
\hline$\left(\mathbf{U}^{(j)}, \mathbf{V}^{(k)}\right)$ & $d_{j} d_{k}$ & $d_{j} d_{k}$ \\
\hline
\end{tabular}

Theorem 5.1: The following three properties are equivalent:

1) $\mathcal{L}$ is weakly proper w.r.t. $\mathcal{E}$.

2) The maximum $s$ - $t$ flow in $\mathcal{N}_{1}$ has value $\sum_{j \in \mathcal{E}_{T}} d_{j}\left(\sum_{k \in \Gamma^{\text {out }[j]}} d_{k}-M_{j}\right)$.

3) The maximum $s$-t flow in $\mathcal{N}_{2}$ has value $\sum_{k \in \mathcal{E}_{R}} d_{k}\left(\sum_{j \in \Gamma^{i n}[k]} d_{j}-N_{k}\right)$.

Proof: By symmetry, we only prove the equivalence of the first two properties. Recall that any subset $S$ of vertices in $\mathcal{N}_{1}$ which contain $s$ but not $t$ defines an $s$ - $t$ cut in in $\mathcal{N}_{1}$, and its capacity is denoted by cut $(S)$. Thus, any $s$ - $t$ cut in $\mathcal{N}_{1}$ consists of $s$, a subset $J$ of $\mathcal{E}_{T}$ and a subset $K$ of $\mathcal{E}_{R}$. Let

$$
\sigma=\sum_{j \in \mathcal{E}_{T}} d_{j}\left(\sum_{k \in \Gamma^{\text {out }}[j]} d_{k}-M_{j}\right) .
$$

For any $J \subseteq \mathcal{E}_{T}$ and $K \subseteq \mathcal{E}_{R}$, let

$$
\begin{aligned}
\delta(J, K)= & \sum_{k \in K} d_{k}\left(N_{k}-d_{k}\right)+\sum_{j \in J} d_{j}\left(M_{j}-d_{j}\right) \\
& -\sum_{(j, k) \in \mathcal{E}[J, K]} d_{k} d_{j} .
\end{aligned}
$$

Consider any $J \subseteq \mathcal{E}_{T}$ and any $K \subseteq \mathcal{E}_{R}$. We claim that

$$
\operatorname{cut}(\{s\} \cup J \cup K)=\sigma+\delta(J, K) .
$$

Indeed,

$$
\begin{aligned}
& c\left(\mathcal{E}\left(J, \mathcal{E}_{R}\right)\right)-\sum_{j \in J} c\left(s, u_{j}\right) \\
& =\sum_{j \in J} d_{j} \sum_{k \in N^{\text {out }}(j)} d_{k}-\sum_{j \in J} d_{j}\left(\sum_{k \in N^{\text {out }}[j]} d_{k}-M_{j}\right) \\
& =\sum_{j \in J} d_{j}\left(M_{j}-d_{j}\right)
\end{aligned}
$$


Therefore,

$$
\begin{aligned}
c u t & (\{s\} \cup J \cup K) \\
= & \sum_{j \in \mathcal{E}_{T} \backslash J} c\left(s, u_{j}\right)+c\left(\mathcal{E}\left[J, \mathcal{E}_{R} \backslash K\right]\right)+\sum_{k \in K} c\left(v_{k}, t\right) \\
= & \sum_{j \in \mathcal{E}_{T}} c\left(s, u_{j}\right)-\sum_{j \in J} c\left(s, v_{j}\right)+c\left(\mathcal{E}\left[J, \mathcal{E}_{R}\right]\right) \\
& -c(\mathcal{E}[J, K])+\sum_{k \in K} c\left(v_{k}, t\right) \\
= & +\sum_{j \in J} d_{j}\left(M_{j}-d_{j}\right)-\sum_{(k, j) \in \mathcal{E}[J, K]} d_{k} d_{j} \\
& +\sum_{k \in K} d_{k}\left(N_{k}-d_{k}\right) \\
= & \sigma+\delta(J, K) .
\end{aligned}
$$

Now, we prove that the first property implies the second property. Suppose that the first property holds. Then, $\delta(J, K) \geq 0$ for any $J \subseteq \mathcal{E}_{T}$ and $K \subseteq \mathcal{E}_{R}$. So, any s-t cut has capacity at least $\sigma$. By the max-flow min-cut theorem, the maximum $s$ - $t$ flow in $\mathcal{N}_{1}$ has value at least $\sigma$.On the other hand, the maximum $s$ - $t$ flow in $\mathcal{N}_{1}$ has value at most $\sigma$. Thus, the second property holds.

Next, we prove that the second property implies the first property. the necessary part. Suppose that the second property holds. By the max-flow min-cut theorem, any $s-t$ has capacity at least $\sigma$. Hence, $\delta(J, K) \geq 0$ for any $J \subseteq \mathcal{E}_{T}$ and $K \subseteq \mathcal{E}_{R}$. So, the first property holds.

In case that all users have a uniform number $d$ of streams, we construct two "slimmed" flow networks $\mathcal{N}_{1}^{\prime}$ and $\mathcal{N}_{2}^{\prime}$ from $\mathcal{N}_{1}$ and $\mathcal{N}_{2}$ respectively by resetting the the arc capacities with the values given in Table II. The next theorem is an immediate consequence of Theorem 5.1 and a simultaneous scaling of flow and capacities.

\begin{tabular}{|c||c|c|}
\hline & $\mathcal{N}_{1}^{\prime}$ & $\mathcal{N}_{2}^{\prime}$ \\
\hline \hline$\left(s, \mathbf{U}^{(j)}\right)$ & $\left|\Gamma^{\text {out }}[j]\right| d-M_{j}$ & $M_{j}-d$ \\
\hline$\left(\mathbf{V}^{(k)}, t\right)$ & $N_{k}-d$ & $\left|\Gamma^{\text {in }}[k]\right| d-N_{k}$ \\
\hline$\left(\mathbf{U}^{(j)}, \mathbf{V}^{(k)}\right)$ & $d$ & $d$ \\
\hline
\end{tabular}
TABLE II. THE ARC CAPACITIES IN THE FLOW NETWORKS $\mathcal{N}_{1}^{\prime}$ AND

Theorem 5.2: Suppose that all users in $\mathcal{L}$ have a uniform number $d$ of streams. Then the following three properties are equivalent:

- $\quad \mathcal{L}$ is weakly proper w.r.t. $\mathcal{E}$.

- The maximum $s$ - $t$ flow in $\mathcal{N}_{1}^{\prime}$ has value $\sum_{j \in \mathcal{E}_{T}}\left(\left|\Gamma^{\text {out }}[j]\right| d-M_{j}\right)$.

- The maximum $s-t$ flow in $\mathcal{N}_{2}^{\prime}$ has value $\sum_{k \in \mathcal{E}_{R}}\left(\left|\Gamma^{i n}[k]\right| d-N_{k}\right)$.

In case that $\mathcal{L}$ is symmetric and $\mathcal{E}$ is complete, we have the following straightforward test of weak properness.

Theorem 5.3: Suppose that $\mathcal{L}=(M, N, d)^{\ell}$ and $\mathcal{E}$ is complete. Then, $\mathcal{L}$ is weakly proper w.r.t. $\mathcal{E}$ if and only if $M+N \geq(\ell+1) d$.
Proof: The necessary part holds trivially from equation (5) with $J=K=[\ell]$. Subsequently, we prove the sufficient part using Theorem 5.2. Consider the flow network $\mathcal{N}_{1}^{\prime} \cdot\left(s, \mathbf{U}^{(j)}\right)$ capacity $\ell d-M,\left(\mathbf{V}^{(k)}, t\right)$ capacity $N-d$ and $\left(\mathbf{U}^{(j)}, \mathbf{V}^{(k)}\right)$ with $j \neq k$ capacity $d$. We construct a (fractional) flow of value $\ell(\ell d-M)$ as follows. First, each arc $\left(s, \mathbf{U}^{(j)}\right)$ carries a flow of value $\ell d-M$, which is its capacity. Then, at each $\mathbf{U}^{(j)}$, its incoming flow is evenly split evenly over the $\ell-1$ arcs leaving itself. In other words, each $\operatorname{arc}\left(\mathbf{U}^{(j)}, \mathbf{V}^{(k)}\right)$ with $j \neq k$ carries a flow of amount $\frac{\ell d-M}{\ell-1}$, which is no more than its capacity $d$ as

$$
\frac{\ell d-M}{\ell-1} \leq \frac{\ell d-d}{\ell-1}=d .
$$

At this moment, the total flow entering each $\mathbf{V}^{(k)}$ has amount

$$
\frac{\ell d-M}{\ell-1}(\ell-1)=\ell d-M \text {. }
$$

This amount of flow is forwarded to $t$ and does not exceed the capacity $N-d$ of the $\operatorname{arc}\left(\mathbf{V}^{(k)}, t\right)$ as $\ell d-M \leq N-d$. Thus, we get a feasible flow of value $\ell(\ell d-M)$. By Theorem 5.2, $\mathcal{L}$ is weakly proper w.r.t. $\mathcal{E}$.

We remark that the special case of the above theorem in which $M=N$ appeared in [3], [27].

\section{B. Properness}

In this subsection, we present flow-based test of properness. We construct two flow networks $\mathcal{N}_{3}$ and $\mathcal{N}_{4}$ as follows. Let $s$ and $t$ be two distinct source and sink vertices. Both networks have the same vertex set

$$
\begin{aligned}
& \{s, t\} \cup\left(\bigcup_{j \in \mathcal{E}_{T}}\left\{\mathbf{U}_{\cdot p}^{(j)}: p \in\left[d_{j}\right]\right\}\right) \cup \\
& \left(\bigcup_{k \in \mathcal{E}_{R}}\left\{\mathbf{V}_{\cdot q}^{(k)}: q \in\left[d_{k}\right]\right\}\right)
\end{aligned}
$$

and the same arc set

$$
\begin{aligned}
& \left(\bigcup_{j \in \mathcal{E}_{T}}\left\{\left(s, \mathbf{U}_{\cdot p}^{(j)}\right): p \in\left[d_{j}\right]\right\}\right) \cup \\
& \left(\bigcup_{k \in \mathcal{E}_{R}}\left\{\left(\mathbf{V}_{\cdot q}^{(k)}, t\right): q \in\left[d_{k}\right]\right\}\right) \cup \\
& \left(\bigcup_{(j, k) \in \mathcal{E}}\left\{\left(\mathbf{U}_{\cdot p}^{(j)}, \mathbf{V}_{\cdot q}^{(k)}\right): p \in\left[d_{j}\right], q \in\left[d_{k}\right]\right\}\right)
\end{aligned}
$$

The arc capacities in these two flow networks are given in Table III. Both flow networks have

$$
2+\sum_{j \in \mathcal{E}_{T}} d_{j}+\sum_{j \in \mathcal{E}_{R}} d_{k}
$$

vertices and

$$
\sum_{j \in \mathcal{E}_{T}} d_{j}+\sum_{j \in \mathcal{E}_{R}} d_{k}+\sum_{(k, j) \in \mathcal{E}} d_{k} d_{j}
$$

arcs. Thus, a maximum $s-t$ flow can be computed in time (cf. Section $10.8 b$ in [24])

$$
\widetilde{O}\left(\left(\sum_{j \in \mathcal{E}_{T}} d_{j}+\sum_{j \in \mathcal{E}_{R}} d_{k}\right)\left(\sum_{(k, j) \in \mathcal{E}} d_{k} d_{j}\right)\right) .
$$

By the max-flow min-cut theorem, the maximum $s$ - $t$ flow in $\mathcal{N}_{3}$ has value at most $\sum_{j \in \mathcal{E}_{T}} d_{j}\left(\sum_{k \in \Gamma^{\text {out }}[j]} d_{k}-M_{j}\right)$, and the maximum $s$ - $t$ flow in $\mathcal{N}_{4}$ has value at most 
$\sum_{k \in \mathcal{E}_{R}} d_{k}\left(\sum_{j \in \Gamma^{i n}[k]} d_{j}-N_{k}\right)$. The theorem below shows that these upper bounds are achievable if and only if $\mathcal{L}$ is proper w.r.t. $\mathcal{E}$. Note that any $s$ - $t$ flow can be fully determined by the flow amount through the $\operatorname{arcs}\left(\mathbf{U}_{\cdot p}^{(j)}, \mathbf{V}_{\cdot q}^{(k)}\right)$ by the flow conservation law.

\begin{tabular}{|c||c|c|}
\hline & $\mathcal{N}_{3}$ & $\mathcal{N}_{4}$ \\
\hline \hline$\left(s, \mathbf{U}_{\cdot p}^{(j)}\right)$ & $\sum_{k \in \Gamma^{\text {out }}[j]} d_{k}-M_{j}$ & $M_{j}-d_{j}$ \\
\hline$\left(\mathbf{V}_{\cdot q}^{(k)}, t\right)$ & $N_{k}-d_{k}$ & $\sum_{j \in \Gamma^{i n}[k]} d_{j}-N_{k}$ \\
\hline$\left(\mathbf{U}_{\cdot p}^{(j)}, \mathbf{V}_{\cdot q}^{(k)}\right)$ & 1 & 1 \\
\hline
\end{tabular}

TABLE III. THE ARC CAPACITIES IN THE FLOW NETWORKS $\mathcal{N}_{3}$ AND
$\mathcal{N}_{4}$.

Theorem 5.4: The following three properties are equivalent:

1) $\mathcal{L}$ is proper w.r.t. $\mathcal{E}$.

2) The maximum $s$ - $t$ flow in $\mathcal{N}_{3}$ has value $\sum_{j \in \mathcal{E}_{T}} d_{j}\left(\sum_{k \in \Gamma^{\text {out }[j]}} d_{k}-M_{j}\right)$.

3) The maximum $s$ - $t$ flow in $\mathcal{N}_{4}$ has value $\sum_{k \in \mathcal{E}_{R}} d_{k}\left(\sum_{j \in \Gamma^{i n}[k]} d_{j}-N_{k}\right)$.

Proof: By symmetry, we only prove the equivalence of the first two properties.

We first prove that first property implies the second property. Suppose the $\mathcal{L}$ is proper w.r.t. $\mathcal{E}$. Let $\mathcal{M}$ be a maximum matching of $G$ in which the largest number of BF variables are matched. We claim that all $\mathrm{BF}$ variables are matched with some equation in $\mathcal{M}$. Assume to the contrary that for some BF block $\mathbf{U}_{\cdot p}^{(j)}$, not all its variables are matched in $\mathcal{M}$. Since the number of equations containing the $\mathrm{BF}$ variables in $\mathbf{U}_{. p}^{(j)}$ is exactly

$\sum_{k \in \Gamma^{\text {out }}(j)} d_{k}=\left(\sum_{k \in \Gamma^{\text {out }}[j]} d_{k}-M_{j}\right)+M_{j}-d_{j}>M_{j}-d_{j}$,

at least one of these equations is matched with a $\mathrm{BF}$ variable. For such equation, we can re-match it with a $\mathrm{BF}$ variable in $\mathbf{U}_{. p}^{(j)}$. The new matching maintained the same size but has matched strictly more $\mathrm{BF}$ variables than $\mathcal{M}$, which is a contradiction. Thus, our claim holds.

From this matching $\mathcal{M}$, we construct an $s-t$ flow by specifying the amount of flow through each $\operatorname{arc}\left(\mathbf{U}_{\cdot p}^{(j)}, \mathbf{V}_{\cdot q}^{(k)}\right)$ : If the equation $f_{p q}^{(j, k)}$ is matched with a $\mathrm{BF}$ variable, then the flow through the arc is one; otherwise, zero. By the flow conservation, the amount of flow through each $\operatorname{arc}\left(s, \mathbf{U}_{\cdot p}^{(j)}\right)$ is exactly the total number of equations containing the $\mathrm{BF}$ variables in $\mathbf{U}_{\cdot p}^{(j)}$ and matched with the BF variables, which is

$$
\sum_{k \in \Gamma^{\text {out }}(j)} d_{k}-\left(M_{j}-d_{j}\right)=\sum_{k \in \Gamma^{\text {out }}[j]} d_{k}-M_{j} .
$$

Similarly, the amount of flow through each $\operatorname{arc}\left(\mathbf{V}_{\cdot q}^{(k)}, t\right)$ is exactly the total number of equations matched with the $\mathrm{ZF}$ variables in $\mathbf{V}_{\cdot q}^{(k)}$, which is at most $N_{k}-d_{k}$. Thus, this flow is feasible, and its total value is exactly $\sum_{j \in \mathcal{E}_{T}} d_{j}\left(\sum_{k \in \Gamma^{\text {out }}[j]} d_{k}-M_{j}\right)$. This completes the necessary part.

Next, we prove that second property implies the first property. Suppose that the second property holds. Since all arc capacities in $\mathcal{N}_{3}$ are integers, there is an integral flow. The amount of flow through each $\operatorname{arc}\left(s, \mathbf{U}_{\cdot p}^{(j)}\right)$ is exactly $\sum_{k \in \Gamma^{\text {out }[j]}} d_{k}-M_{j}$, and the amount of flow through each $\operatorname{arc}\left(\mathbf{U}_{\cdot p}^{(j)}, \mathbf{V}_{\cdot q}^{(k)}\right)$ is either zero or one. The flow induces a matching scheme as follows: Each equation $f_{p q}^{(j, k)}$ is matched with a ZF (respectively, BF) variable if the amount of flow through the $\operatorname{arc}\left(\mathbf{U}_{\cdot p}^{(j)}, \mathbf{V}_{\cdot q}^{(k)}\right)$ is one (respectively, zero). Under this scheme, the number of equations which contain the $\mathrm{BF}$ variables in $\mathbf{U}_{. p}^{(j)}$ but are matched with the $\mathrm{ZF}$ variables is exactly the amount of flow leaving (and equivalently, entering) $\mathbf{U}_{. p}^{(j)}$, which is exactly $\sum_{k \in \Gamma^{\text {out }}[j]} d_{k}-M_{j}$. Thus, the number of equations expected to be matched with the BF variables in $\mathbf{U}_{. p}^{(j)}$ is exactly

$$
\sum_{k \in \Gamma^{\text {out }}(j)} d_{k}-\left(\sum_{k \in \Gamma^{\text {out }}[j]} d_{k}-M_{j}\right)=M_{j}-d_{j},
$$

Similarly, the number of equations expected to be matched with the ZF variables in $\mathbf{V}_{\cdot q}^{(k)}$ is exactly the amount of flow entering (and equivalently, leaving) $\mathbf{V}_{\cdot q}^{(k)}$, which is at most $N_{k}-d_{k}$. Thus, such matching scheme is realizable.

In case that all users have a uniform number of streams, the next theorem reveals the equivalence of weaker properness and properness.

Theorem 5.5: Suppose that all users in $\mathcal{L}$ have a uniform number $d$ of streams. Then, $\mathcal{L}$ is proper w.r.t. $\mathcal{E}$ if and only if $\mathcal{L}$ is weakly proper w.r.t. $\mathcal{E}$.

Proof: The necessary part is trivial, and we only prove the sufficient part. Suppose that $\mathcal{L}$ is weakly proper w.r.t. $\mathcal{E}$. By Theorem 5.2, the flow network $\mathcal{N}_{1}^{\prime}$ has an integral $s$ - $t$ flow of value $\sum_{j \in \mathcal{E}_{T}}\left(\left|\Gamma^{\text {out }}[j]\right| d-M_{j}\right)$. Let $y_{j k}$ be the amount of this flow though $\left(\mathbf{U}^{(j)}, \mathbf{V}^{(k)}\right)$ in $\mathcal{N}_{1}^{\prime}$. Then, $y_{j k} \leq d$. By the flow conservation law,

$$
\begin{aligned}
\sum_{j \in \Gamma^{\text {in }}(k)} y_{j k} & \leq N_{k}-d, \forall k \in \mathcal{E}_{R} ; \\
\sum_{k \in N^{\text {out }}(j)} y_{j k} & =\left|\Gamma^{\text {out }}[j]\right| d-M_{j}, \forall j \in \mathcal{E}_{T} .
\end{aligned}
$$

We shall construct an $s$ - $t$ flow in $\mathcal{N}_{3}$ by specifying the amount of flow through each $\operatorname{arc}\left(\mathbf{U}_{\cdot p}^{(j)}, \mathbf{V}_{\cdot q}^{(k)}\right)$ below.

Consider any $(j, k) \in \mathcal{E}$. For each $l \in\left[y_{j k}\right]$, we define a perfect matching between $\left\{\mathbf{U}_{\cdot p}^{(j)}: p \in[d]\right\}$ and $\left\{\mathbf{V}_{\cdot q}^{(k)}: q \in[d]\right\}$ by

$$
\left\{\left(\mathbf{U}_{\cdot p}^{(j)}, \mathbf{V}_{\cdot q}^{(k)}\right): p \in[d], q=(p+l-1) \bmod d+1\right\} .
$$

Since $y_{j k} \leq d$, the union of these $y_{j k}$ perfect matching is a $y_{j k}$-regular bipartite graph $G_{j k}$ on

$$
\left\{\mathbf{U}_{\cdot p}^{(j)}: p \in[d]\right\} \cup\left\{\mathbf{V}_{\cdot q}^{(k)}: q \in[d]\right\} .
$$


Then, the amount of flow in $\mathcal{N}_{3}$ through the $\operatorname{arc}\left(\mathbf{U}_{\cdot p}^{(j)}, \mathbf{V}_{\cdot q}^{(k)}\right)$ is one if $\mathbf{U}_{\cdot p}^{(j)}$ and $\mathbf{V}_{\cdot q}^{(k)}$ are adjacent in $G_{j k}$, and zero otherwise. We verify that such flow is feasible and has value exactly $\sum_{j \in \mathcal{E}_{T}}\left(\left|\Gamma^{\text {out }}[j]\right| d-M_{j}\right)$.

Since $G_{j k}$ is $y_{j k}$-regular, the total amount of flow leaving $\mathbf{U}_{\cdot p}^{(j)}$ for $\left\{\mathbf{V}_{\cdot q}^{(k)}: q \in[d]\right\}$ is exactly $y_{j k}$. So, the total amount of flow leaving $\mathbf{U}_{\cdot p}^{(j)}$ is exactly

$$
\sum_{k \in \Gamma^{\text {out }}(j)} y_{j k}=d\left|\Gamma^{\text {out }}[j]\right|-M_{j} .
$$

By the flow conservation, this is also the exact amount of flow through each arc $\left(s, \mathbf{U}_{\cdot p}^{(j)}\right)$. Similarly, the total amount of flow entering $\mathbf{V}_{\cdot q}^{(k)}$ from $\left\{\mathbf{U}_{\cdot p}^{(j)}: p \in[d]\right\}$ is exactly $y_{j k}$, and the total amount of flow leaving $\mathbf{V}_{\cdot q}^{(k)}$ is exactly

$$
\sum_{j \in \Gamma^{i n}\{k\}} y_{j k} \leq N_{k}-d .
$$

By the flow conservation, the amount of flow through the arc $\left(\mathbf{V}_{\cdot q}^{(k)}, t\right)$ is $N_{k}-d$. Thus, the flow is feasible. The value of this flow, which is the total amount of flow leaving $s$, is exactly $\sum_{j \in \mathcal{E}_{T}}\left(\left|\Gamma^{\text {out }}[j]\right| d-M_{j}\right)$. By Theorem 5.5 , the systems is proper.

The above theorem and Theorem 5.3 yields the following corollary immediately.

Corollary 5.6: Suppose that $\mathcal{L}=(M, N, d)^{\ell}$ and $\mathcal{E}$ is complete. Then, the following three properties are equivalent:

1) $\mathcal{L}$ is proper w.r.t. $\mathcal{E}$.

2) $\mathcal{L}$ is weakly proper w.r.t. $\mathcal{E}$.

3) $\quad M+N \geq(\ell+1) d$.

The equivalence of the first and the third properties in the above corollary first appeared as Theorem 1 in [29] and was utilized to derive several important corollaries. However, [29] only gives the proof of the (easier) implication of the third property from the first property. The reverse direction was left unproven.

\section{Strong Properness}

The test of strong properness appears to be intractable in general. In this subsection, we present a flow-based test of strong properness in the uniform-stream setting. Suppose that all users in $\mathcal{L}$ have a uniform number $d$ of streams. We construct two flow networks $\mathcal{N}_{5}$ and $\mathcal{N}_{6}$ from $\mathcal{N}_{1}$ and $\mathcal{N}_{2}$ respectively by resetting the the arc capacities with the values given in Table IV. By the max-flow min-cut theorem, the maximum $s$ - $t$ flow in $\mathcal{N}_{5}$ has value at most $\sum_{j \in \mathcal{E}_{T}}\left(\left|\Gamma^{\text {out }}[j]\right| d-M_{j}\right)$, and the maximum $s$ - $t$ flow in $\mathcal{N}_{6}$ has value at most $\sum_{k \in \mathcal{E}_{R}}\left(\left|\Gamma^{i n}[k]\right| d-N_{k}\right)$.

Theorem 5.7: The following two statements hold:

1) $\mathcal{L}$ is $\mathrm{Tx}$-strongly proper w.r.t. $\mathcal{E}$ if and only if the maximum $s$ - $t$ flow in $\mathcal{N}_{5}$ is $\sum_{j \in \mathcal{E}_{T}}\left(\left|\Gamma^{\text {out }}[j]\right| d-M_{j}\right)$.

2) $\mathcal{L}$ is $\mathrm{Rx}$-strongly proper w.r.t. $\mathcal{E}$ if and only if the maximum $s$ - $t$ flow in $\mathcal{N}_{6}$ is $\sum_{k \in \mathcal{E}_{R}}\left(\left|\Gamma^{i n}[k]\right| d-N_{k}\right)$.

\begin{tabular}{|c||c|c|}
\hline & $\mathcal{N}_{5}$ & $\mathcal{N}_{6}$ \\
\hline \hline$\left(s, \mathbf{U}^{(j)}\right)$ & $d\left|\Gamma^{\text {out }}[j]\right| d-M_{j}$ & {$\left[\frac{M_{j}}{d}\right] d-d$} \\
\hline$\left(\mathbf{V}^{(k)}, t\right)$ & {$\left[\frac{N_{k}}{d}\right] d-d$} & $\left|\Gamma^{i n}[k]\right| d-N_{k}$ \\
\hline$\left(\mathbf{U}^{(j)}, \mathbf{V}^{(k)}\right)$ & $d$ & $d$ \\
\hline
\end{tabular}

TABLE IV. THE ARC CAPACITIES IN THE FLOW NETWORKS $\mathcal{N}_{5}$ AND $\mathcal{N}_{6}$.

Proof: By symmetry, we only prove the first statement.

We first prove the necessary part. Suppose that $\mathcal{L}$ is $\mathrm{Tx}-$ strongly proper w.r.t. $\mathcal{E}$. Let $\mathcal{M}$ be a maximum Tx-strong matching in which the largest number of $\mathrm{BF}$ variables are matched. Since $\mathcal{M}$ is Tx-strong, for any $j$ all of the BF block $\mathbf{U}_{\cdot p}^{(j)}$ for $p \in[d]$ have the same number of matched variables, which is denoted by $\tau_{j}$. We claim that all $\mathrm{BF}$ variables are matched in $\mathcal{M}$; in other words, $\tau_{j}=M_{j}-d$ for each $j$. Assume to the contrary that for some $j, \tau_{j}<M_{j}-d$. Since the number of equations containing the $\mathrm{BF}$ variables in $\mathbf{U}_{\cdot 1}^{(j)}$ is exactly

$$
\left|\Gamma^{\text {out }}(j)\right| d=\left(\left|\Gamma^{\text {out }}[j]\right| d-M_{j}\right)+M_{j}-d>M_{j}-d,
$$

at least one of these equations is matched with a ZF variable. Let $f_{1 q}^{(j, k)}$ be a such equation. Since $\mathcal{M}$ is TX-strong, all of the $d$ equations in the group $\mathbf{F}_{\cdot q}^{(j, k)}$ are matched with ZF variables. For each $p \in[d]$, we can re-match $f_{p q}^{(j, k)}$ with a BF variable in $\mathbf{U}_{. p}^{(j)}$ which is unmatched in $\mathcal{M}$. The new matching maintains the same size and is still Tx-strong; but it has matched strictly more $\mathrm{BF}$ variables than $\mathcal{M}$, which is a contradiction. Thus, our claim holds.

From the matching $\mathcal{M}$, we construct an $s$ - $t$ flow in in $\mathcal{N}_{5}$ by specifying the amount of flow through each arc $\left(\mathbf{U}^{(j)}, \mathbf{V}^{(k)}\right)$ as follows. For any $(j, k) \in \mathcal{E}$ and $q \in[d]$, let $x_{j k q}=1$ if the group $\mathbf{F}_{\cdot q}^{(j, k)}$ of equations are matched with ZF variables, and $x_{j k q}=0$ otherwise. In addition, let $x_{j k}=\sum_{q \in\left[d_{k}\right]} x_{j k q}$. Clearly, $x_{j k} \leq d$. The amount of flow through the arc $\left(\mathbf{U}^{(j)}, \mathbf{V}^{(k)}\right)$ is exactly $x_{j k}$. By the flow conservation, the total flow through the $\operatorname{arc}\left(s, \mathbf{U}^{(j)}\right)$ is exactly $\sum_{k \in \Gamma^{\text {out }}(j)} x_{j k}$, and the total flow through the $\operatorname{arc}\left(\mathbf{V}^{(k)}, t\right)$ is exactly $\sum_{k \in \Gamma_{\text {out }}(j)} x_{j k}$. We shall show that

$$
\begin{gathered}
\sum_{k \in \Gamma^{\text {out }}(j)} x_{j k}=\left|\Gamma^{\text {out }}[j]\right| d-M_{j}, \forall j \in \mathcal{E}_{T} ; \\
\sum_{j \in \Gamma^{\text {in }}(k)} x_{j k} \leq d\left(\left\lfloor\frac{N_{k}}{d}\right\rfloor-1\right), \forall k \in \mathcal{E}_{R} .
\end{gathered}
$$

These properties yields that the flow is feasible and its value is the maximum possible one given by $\sum_{j \in \mathcal{E}_{T}}\left(\left|\Gamma^{\text {out }}[j]\right| d-M_{j}\right)$.

Consider any $j \in \mathcal{E}_{T}$ and any $p \in[d]$. On one hand, the the total number of matched variables in $\mathbf{U}_{\cdot p}^{(j)}$ is exactly $M_{j}-d$. On the other hand, the total number of matched variables in $\mathbf{U}_{. p}^{(j)}$ is exactly

$$
\begin{aligned}
& \sum_{k \in \Gamma^{\text {out }}(j)} \sum_{q \in[d]}\left(1-x_{j k q}\right)=\left|\Gamma^{\text {out }}(j)\right| d-\sum_{k \in \Gamma^{\text {out }}(j)} \sum_{q \in[d]} x_{j k q} \\
= & \left|\Gamma^{\text {out }}(j)\right| d-\sum_{k \in \Gamma^{\text {out }}(j)} x_{j k} .
\end{aligned}
$$


Thus,

$$
\left|\Gamma^{\text {out }}(j)\right| d-\sum_{k \in \Gamma^{o u t}(j)} x_{j k}=M_{j}-d,
$$

which implies that

$$
\sum_{k \in \Gamma^{\text {out }}(j)} x_{j k}=d\left|\Gamma^{\text {out }}[j]\right|-M_{j} .
$$

Consider any $k \in \mathcal{E}_{R}$ and any $q \in[d]$. For any $j \in \Gamma^{i n}(k)$ with $x_{j k q}=1$, all the $d$ equations in the group $\mathbf{F}_{\cdot q}^{(j, k)}$ are matched with ZF variables of $\mathbf{V}_{\cdot q}^{(k)}$. Thus, the total number of matched variables in $\mathbf{V}_{\cdot q}^{(k)}$ is

$$
\sum_{j \in \Gamma^{i n}(k)} x_{j k q} d \leq N_{k}-d,
$$

which implies

$$
\sum_{j \in \Gamma^{i n}(k)} x_{j k q} \leq \frac{N_{k}}{d}-1 .
$$

Since the left-hand side is an integer, we further have

$$
\sum_{j \in \Gamma^{i n}(k)} x_{j k q} \leq\left\lfloor\frac{N_{k}}{d}\right\rfloor-1 .
$$

Thus,

$$
\begin{aligned}
\sum_{j \in \Gamma^{i n}(k)} x_{j k} & =\sum_{j \in \Gamma^{i n}(k)} \sum_{q \in[d]} x_{j k q}=\sum_{q \in[d]} \sum_{j \in \Gamma^{i n}(k)} x_{j k q} \\
& \leq d\left(\left\lfloor\frac{N_{k}}{d}\right\rfloor-1\right) .
\end{aligned}
$$

Next, we prove the sufficient part. Suppose that the second property holds. Suppose that the maximum $s$ - $t$ flow in $\mathcal{N}_{5}$ is exactly $\sum_{j \in \mathcal{E}_{T}}\left(\left|\Gamma^{\text {out }}[j]\right| d-M_{j}\right)$. Since all arc capacities in $\mathcal{N}_{5}$ are integers, there is an integral $s$ - $t$ flow of the same value. Let $x_{j k}$ be the amount of this integral flow through the arc $\left(\mathbf{U}^{(j)}, \mathbf{V}^{(k)}\right)$; clearly, $x_{j k} \leq d$. In addition, by the flow conservation,

$$
\begin{gathered}
\sum_{k \in \Gamma^{\text {out }}(j)} x_{j k}=d\left|\Gamma^{\text {out }}[j]\right|-M_{j}, \forall j \in \mathcal{E}_{T} ; \\
\sum_{j \in \Gamma^{\text {in }}(k)} x_{j k} \leq d\left(\left\lfloor\frac{N_{k}}{d}\right\rfloor-1\right), \forall k \in \mathcal{E}_{R} .
\end{gathered}
$$

For each $(k, j) \in \mathcal{E}$, a decomposition of $x_{j k}$ consists of

$$
\left\{x_{j k q} \in\{0,1\}: q \in[d]\right\}
$$

satisfying that

$$
\sum_{q \in[d]} x_{j k q}=x_{j k}
$$

For any $k \in \mathcal{E}_{R}$, we apply the algorithm $\operatorname{Decompose}(k)$ illustrated in Table $\mathrm{V}$ to jointly compute a decomposition of $x_{j k}$ for each $j \in \Gamma^{i n}(k)$ satisfying that

$$
\begin{aligned}
\max _{q \in[d]} \sum_{j \in \Gamma^{i n}(k)} x_{j k q} & \leq\left\lceil\frac{\sum_{j \in \Gamma^{i n}(k)} x_{j k}}{d}\right\rceil \leq\left\lceil\frac{d\left(\left\lfloor\frac{N_{k}}{d}\right\rfloor-1\right)}{d}\right\rceil \\
& =\left\lfloor\frac{N_{k}}{d}\right\rfloor-1 .
\end{aligned}
$$

These decompositions naturally induce a Tx-strong matching scheme as follows: For each $(k, j) \in \mathcal{E}$ and each $q \in[d]$, all of the $d$ equations in the group $\mathbf{F}_{\cdot q}^{(j, k)}$ are matched with $\mathrm{ZF}$ variables if $x_{j k q}=1$, and with the $\mathrm{BF}$ variables otherwise. Under this scheme, the number of equations expected to be matched with the $\mathrm{BF}$ variables in $\mathbf{U}_{\cdot p}^{(j)}$ is exactly

$$
\begin{aligned}
& \sum_{k \in \Gamma_{\text {out }}(j)} \sum_{q \in[d]}\left(1-x_{j k q}\right) \\
= & \left|\Gamma^{\text {out }}(j)\right| d-\sum_{k \in \Gamma^{\text {out }}(j)} \sum_{q \in[d]} x_{j k q} \\
= & \left|\Gamma^{\text {out }}(j)\right| d-\sum_{k \in \Gamma^{\text {out }}(j)} x_{j k} \\
= & \left|\Gamma^{\text {out }}(j)\right| d-\left(d\left|\Gamma^{\text {out }}[j]\right|-M_{j}\right) \\
= & M_{j}-d ;
\end{aligned}
$$

and the number of equations expected to be matched with the $\mathrm{ZF}$ variables in each $\mathbf{V}_{\cdot q}^{(k)}$ is exactly

$$
\sum_{j \in \Gamma^{i n}(k)} x_{j k q} d \leq d\left(\left\lfloor\frac{N_{k}}{d}\right\rfloor-1\right) \leq N_{k}-d .
$$

Thus, such matching scheme is realizable.

TABLE V.

$$
\begin{aligned}
& \hline \text { Algorithm Decompose }(k) \\
& \hline \hline \text { for each } j \in \Gamma^{i n}(k) \text { do } \\
& \text { for each } q=1 \text { to } d \text { do } x_{j k q} \leftarrow 0 ; \\
& q \leftarrow 0 \text {; } \\
& \text { for each } j \in \Gamma^{i n}(k) \text { do } \\
& \text { for } i=1 \text { to } x_{j k} \text { do } \\
& \qquad \leftarrow(q \bmod d)+1 ; \\
& x_{j k q} \leftarrow 1 ; \\
& \text { Output }\left\{x_{j k q}: j \in \Gamma^{i n}(k), q \in[d]\right\} .
\end{aligned}
$$

OUTLINE OF THE ALGORITHM Decomposition $(k)$.

Note that when $d \mid N_{k}$ for each $k \in \mathcal{E}_{R}$, the two networks $\mathcal{N}_{5}$ and $\mathcal{N}_{1}$ (respectively, $\mathcal{N}_{6}$ and $\mathcal{N}_{2}$ ) are identical. Similarly, Thus, when $d \mid M_{j}$ for each $j \in \mathcal{E}_{T}$, the two networks $\mathcal{N}_{6}$ and $\mathcal{N}_{2}$ are identical. Thus, Theorem 5.1 and Theorem 5.7 immediately yields the following theorem.

Theorem 5.8: Suppose that either $d \mid N_{k}$ for each $k \in \mathcal{E}_{R}$, or $d \mid M_{j}$ for each $j \in \mathcal{E}_{T}$. Then $\mathcal{L}$ is strongly proper w.r.t. $\mathcal{E}$ if and only if $\mathcal{L}$ is weakly proper w.r.t. $\mathcal{E}$. Consequently, the feasibility, strong properness, properness, and weak properness of $\mathcal{L}$ w.r.t. $\mathcal{E}$ are all equivalent.

An immediate consequence of the above theorem is that for the single-stream user instances, the feasibility and weak properness of any the single-stream user instance w.r.t. any interference topology are equivalent.

\section{SUMMARY}

In this paper, we introduced three variants of properness of an arbitrary user instance $\mathcal{L}$ w.r.t. an arbitrary interference topology $\mathcal{E}$. The relations among them and the (generic LIA) feasibility are illustrated in Figure 1. The solid arrows represents the implications without any condition. The dashed arrows represent the implications with additional conditions. Specifically, If all users have uniform streams, then weak 
properness implies properness. If all user have a uniform number of streams which either divides the number of transmitting antennas of every user or divides the number of receiving antennas of every user, then weak properness implies strong properness. We also introduced an reduction operation on the interference topology which preserves all four properties. By a sequence of reductions, an irreducible interference topology can be obtained from $\mathcal{E}$. After this simple preprocessing, the weak properness, properness, and strong properness (with uniform streams) can be tested by the fast and robust flowbased testing algorithms.

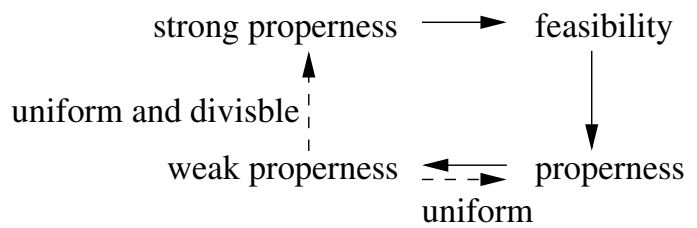

Fig. 1. The relations among strong properness, feasibility, properness, and weak properness.

We conclude this paper with two open problems for the future studies. The first major open problem is the computational complexity of the feasibility test. The second one is the following conjecture on symmetric user instances: For $\mathcal{L}=(M, N, d)^{\ell}$ with $2 d \leq \min \{M, N\}$ and an arbitrary interference topology $\mathcal{E}$, the feasibility and properness are equivalent. Note that the correctness of this conjecture with a complete interference topology was asserted in [23].

ACKNOWLEDGEMENTS: This work was supported in part by the National Science Foundation of USA under grants CNS-1454770, CNS-1219109, and CNS-1217309, by the National Natural Science Foundation of P. R. China under grant 61272524 , and by Dalian University of Technology under the Haitian Scholar grant.

\section{REFERENCES}

[1] O. El Ayach, S. Peters, and R. W. J. Heath, The feasibility of interference alignment over measured MIMO-OFDM channels, IEEE Trans. on Vehicular Technology 59:4309-4321, 2010.

[2] L. Blum, F. Cucker, M. Shub, and S. Smale, Complexity and Real Computation. New York: Springer-Verlag, 1998.

[3] G. Bresler, D. Cartwright, and D. Tse, Settling the feasibility of interference alignment for the MIMO interference channel: the symmetric square case, ArXiv preprint available at http://arxiv.org/abs/1104.0888v1, 2011.

[4] V. R. Cadambe and S. A. Jafar, Interference alignment and degrees of freedom region of the $k$-user interference channel, IEEE Trans. on Inf. Theory 54(8):3425-3441, 2008.

[5] Z. Chen and A. Storjohann, A BLAS based C library for exact linear algebra on integer matrices, in Proc. ISSAC 2005, pp. 92-99.

[6] J.-G. Dumas, T. Gautier, M. Giesbrecht, P. Giorgi, B. Hovinen, E. Kaltofen, et al., J.-G. Dumas, T. Gautier, M. Giesbrecht, P. Giorgi, B. Hovinen, E. Kaltofen, B.D. Saunders, W. J. Turner, and, G. Villard, LinBox: A generic library for exact linear algebra, in Proc. 1st ICMS 2002, pp. 40-50.

[7] S. Gollakota, S. D. Perli, and D. Katabi, Interference alignment and cancellation, SIGCOMM Comput. Commun. Rev. 39:159-170, 2009.

[8] Ó. González, C. Beltrán, and I. Santamaría, A Feasibility fest for linear interference alignment in MIMO channels with constant coefficients, IEEE Transactions on Information Theory 60(3):1840-1856, 2014 Conference version appeared in IEEE International Symposium on Information Theory (ISIT) 2012
[9] O. Gonzalez, D. Ramirez, I. Santamaria, J. A. Garcia-Naya, and L. Castedo, Experimental validation of interference alignment techniques using a multiuser MIMO testbed, in Proc. International ITG Workshop on Smart Antennas (WSA) 2011.

[10] M. Guillaud, and D. Gesbert, Interference Alignment in Partially Connected Interfering Multiple-Access and Broadcast Channels, in Proc. IEEE Global Telecommunications Conference (GLOBECOM) 2011

[11] H. Huang, and V. Lau, Partial Interference Alignment for K-user MIMO Interference Channels, IEEE Transactions on Signal Processing 59(10): 4900-4908, 2011.

[12] S. A. Jafar, Interference alignment: A new look at signal dimensions in a communication network, Foundations and Trends in Communications and Information Theory 7(1):1-136, 2011.

[13] S. A. Jafar and S. Shamai, Degrees of freedom region of the MIMO X channel, IEEE Trans. Inf. Theory 54(1):151-170, 2008.

[14] N. Lee, D. Park, and Y.-D. Kim, Degrees of freedom on the Kuser MIMO interference channel with constant channel coefficients for downlink communications, in Proc. IEEE Global Telecommunications Conference (Globecom) 2009.

[15] Y.-F. Liu, Y.-H. Dai, and Z.-Q. Luo, On the complexity of leakage interference minimization for interference alignment, in Proc. IEEE Int'l Workshop on Signal Processing Advances on Wireless Communications (SPAWC) 2010.

[16] M. Maddah-Ali, A. Motahari, and A. Khandani, Communications over MIMO X channels: Interference alignment, decomposition, and performance analysis, IEEE Trans. on Inf. Theory 54(8):3457-3470, 2008.

[17] C. D. Meyer, Matrix Analysis and Applied Linear Algebra, 1st ed., SIAM, 2000.

[18] F. Negro, S. P. Shenoy, I. Ghauri, and D. T. M. Slock, Interference alignment feasibility in constant coefficient MIMO interference channels, in Proc. IEEE Int'l Workshop on Signal Processing Advances on Wireless Communications (SPAWC) 2010.

[19] M. Razaviyayn, M. S. Boroujeni, and Z.-Q. Luo, Linear transceiver design for interference alignment: Complexity and computation, ArXiv preprint available: http://arxiv.org/abs/1009.3481, 2010.

[20] M. Razaviyayn, G. Lyubeznik, and Z.-Q. Luo, On the degrees of freedom achievable through interference alignment in a MIMO interference channel, IEEE Trans. on Signal Processing 60(2):812-821, 2012.

[21] L. Ruan and V. Lau, Dynamic interference mitigation for generalized partially connected quasi-static MIMO interference channel, IEEE Trans. on Signal Processing 59(8): 3788-3798, 2011.

[22] L. Ruan, V. Lau, and X. Rao, Interference alignment for partiallyconnected MIMO cellular networks, IEEE Trans. on Signal Processing 60(7): 3692-3701, 2012.

[23] L. Ruan, V. Lau, and M. Z. Win, The Feasibility Conditions for Interference Alignment in MIMO Networks, IEEE Transactions on Signal Processing 61(8): 2066-2077, 2013. Conference version appeared in IEEE International Symposium on Information Theory (ISIT) 2012: 2496-2500.

[24] A. Schrijver, Combinatorial optimization: polyhedra and efficiency, Springer, 2003.

[25] M. Shub and S. Smale, Complexity of Bézout's theorem. I. Geometric aspects, J. Amer. Math. Soc., vol. 6, no. 2, pp. 459-501, 1993.

[26] M. Sipser, Introduction to the Theory of Computation. PWS Publishing, 1997.

[27] R. Srinivasan, D. Blough, and P. Santi, Optimal One-Shot Stream Scheduling for MIMO Links in a Single Collision Domain, in Proc. IEEE SECON 2009: 1-9.

[28] M. Westreicher and M. Guillaud, Interference alignment over partiallyconnected interference networks: Application to the cellular case, in Proc. IEEE WCNC. 2012

[29] C. M. Yetis, T. Gou, S. A. Jafar, and A. H. Kayran, On feasibility of interference alignment in MIMO interference networks, IEEE Trans. on Signal Processing 58(9):4771-4782, 2010. 